\title{
Life History Phenology and Sediment Size Association of the Dragonfly Cordulegaster dorsalis (Odonata: Cordulegastridae) in an Ephemeral Habitat in Southwestern British Columbia
}

\author{
Laurie B. MarczaK ${ }^{1}$, John S. Richardson ${ }^{1}$, and Marie-Claire Classen ${ }^{2}$
}

${ }^{1}$ Department of Forest Sciences, University of British Columbia, 3041-2424 Main Mall, Vancouver, British Columbia V6T 1Z4 Canada; Corresponding author: e-mail: laurie@interchange.ubc.ca

${ }^{2}$ Department of Biological Sciences, University of Alberta, CW405, Biological Sciences Centre, University of Alberta, Edmonton, Alberta T6G 2E9 Canada

Marczak, Laurie B., John S. Richardson, and Marie-Claire Classen. 2006. Life history phenology and sediment size association of the dragonfly Cordulegaster dorsalis (Odonata: Cordulegastridae) in an ephemeral habitat in southwestern British Columbia. Canadian Field Naturalist 120(3): 347-350.

The life cycle of the dragonfly Cordulegaster dorsalis was studied over one year by systematic sampling of larvae in three intermittent headwater streams in southwestern British Columbia. We determined that larvae normally take three years to reach maturity, emerging throughout July and August. There is limited evidence suggesting a split cohort development, with early emergence after two years. Additionally, we tested whether larval instars were distributed randomly or if they occupied different sediment microhabitats. Smaller animals tend to be associated with smaller grained organic sediments, although there was high variation between the streams.

Key Words: Odonata, dragonfly, Cordulegaster dorsalis, ephemeral stream, life history, phenology, British Columbia.

Dragonfly larvae are often conspicuous and important predators in stream ecosystems. In British Columbia, the family Cordulegastridae is represented by a single species, Cordulegaster dorsalis Hagen. Cordulegastrids occur in lotic waters, usually in small streams, where larvae live as shallow burrowers in sediment (Cannings 2002). In southwestern coastal British Columbia, larvae of $C$. dorsalis are often found in small headwater streams that are subject to extreme episodic drying. In the interior of the province, the species is less common and is mainly associated with small, spring-fed streams. Adults of $C$. dorsalis normally fly from mid-May to early September, peaking in July (Cannings 2002). Published accounts of the life history, habitat associations or ecological requirements of $C$.dorsalis in its larval stage are sparse.

Kennedy (1917) estimated $C$. dorsalis larvae in central California may require as many as four years to develop. The larval development of cordulegastrids in Europe ranges from 2 to 5 years and may include split cohorts (Ferreras-Romero and Corbet 1999). Split cohort timing has been documented in other species in the genus (C. boltonii [Donovan] in Ferreras-Romero and Corbet 1999). Corbet (1999: 230) maintains it is possible to identify a winter critical size, below which larvae in the first year of development will enter diapause during the subsequent summer period, requiring another year's growth to reach metamorphosis. Such differential growth rates within a single cohort may be produced by staggered egg-laying times, food limitation or variability in the environment. Given the tran- sitory nature of the streams used by $C$. dorsalis, it is possible that it exhibits plasticity in growth rates and emergence times in response to continual shrinking and rewetting of the streams.

Dragonfly larvae increase in size by three to four orders of magnitude as they develop. Microhabitat selection may have much to do with the physical constraints of size. Different species of burrowing odonates prefer certain sediment-size categories (Corbet 1999). C. boltonii has sediment-size preferences that vary with specific larval size classes (Prodon 1976 unpublished dissertation referenced in Corbet 1999). In contrast, C. maculata Sélys in Virginia is a habitat generalist, occuring in equal densities in both silts and sands (Burcher and Smock 2002). In general, small larvae of $C$. boltonii prefer much finer sediments than do larger larvae - larvae tend to select a particle size that offers the least obstruction to burrowing (Corbet 1999).

We report life-history observations (morphological measurements) of larval $C$. dorsalis over a one year period in three small headwater streams subject to late summer drying. We observed that large and small larvae did not occur together within pools. At the same time, we observed surprisingly high densities of odonate larvae in small, shrinking pools during late summer. We tested whether small and large larval instars occupy different sediment microhabitats and report lifehistory observations (morphological measurements) of larval $C$. dorsalis over a one-year period in three small headwater streams subject to late summer drying. 


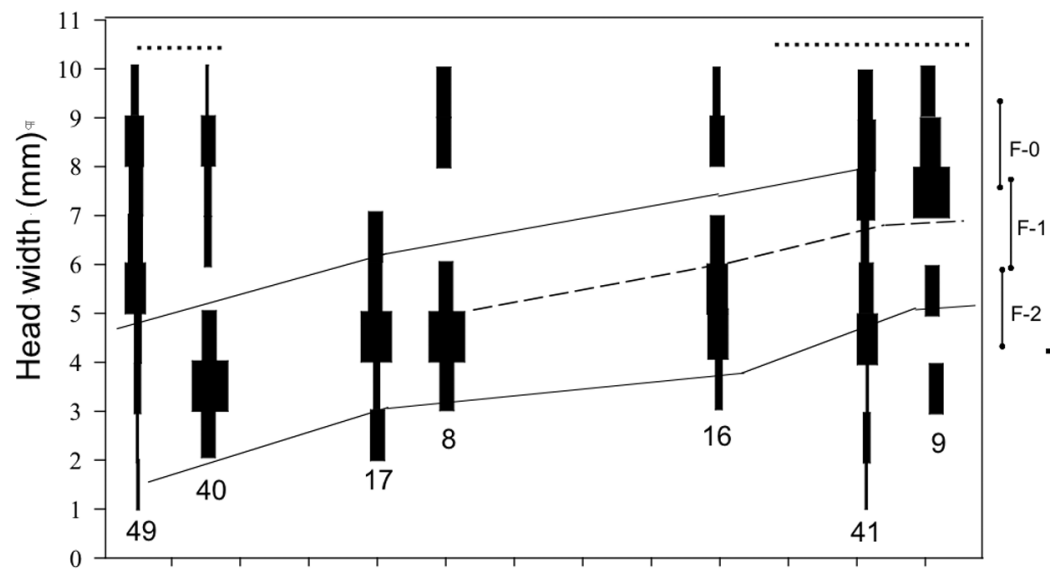

Aug Sep Oct Nov Dec Jan Feb Mar Apr May Jun Jul

FIGURE 1. Life history patterns of Cordulegaster dorsalis shown for 2002-2003 - in G Creek $(n=175)$. Continuous lines ascending from left to right indicate inferred boundaries between successive hatching cohorts. The emergence period during late summer is shown (dotted line). Size ranges of the last three instars are indicated. The broken line indicates an alternate interpretation and represents the inferred boundary between the two components that could result from cohort-splitting in the two-year-old hatching cohort, thus distinguishing larvae that grow fast (above line) and slowly (below line). Numbers below columns indicate sample sizes for each sampling date. Widths of black bars for each interval of headwidth on a given date represent the relative fraction of the total sample that fell in that interval; total possible width for any given sampling date is represented by the black legend box labelled $100 \%$.

\begin{abstract}
Methods
Study Site

This study took place in the 5157 ha Malcolm Knapp Research Forest, located in Maple Ridge, British Columbia. The forest is located in the Pacific coastal rain forest, with a maritime climate of cool dry summers and warm wet winters (Naiman et al. 2000). We sampled Cordulegaster dorsalis larvae for life history characteristics and sediment associations in three streams within the forest (I, F and G streams; $49^{\circ} 18^{\prime} 40^{\prime \prime} \mathrm{N}, 122^{\circ} 32^{\prime} 40^{\prime \prime} \mathrm{W}$ ), which were part of an ongoing riparian management experiment (see Kiffney et al. (2003) for site descriptions). Both F and G streams retained a $10 \mathrm{~m}$ forest buffer while I stream flowed through a 5-year-old clearcut. The dominant vegetation surrounding these creeks included Red Alder (Alnus rubra) and Vine Maple (Acer circinatum) with a canopy composed largely of Western Hemlock (Tsuga heterophylla), Western Redcedar (Thuja plicata) and Douglas-fir (Pseudotsuga menziesii). Vegetation adjacent to I stream consisted of shrubs, particularly Salmonberry (Rubus spectabilis) and Huckleberry (Vaccinium ovatum).
\end{abstract}

\section{Life-history characteristics}

We surveyed approximately $200 \mathrm{~m}$ each of I, F, and $\mathrm{G}$ streams in August, October and December of 2002 and April, July and August of 2003, collecting larvae using dip nets and hand sorting. We measured head width (Hdw max. distance between the lateral margins of the compound eyes), total body length ( $\mathrm{Tbl}$; max. distance between the mouthparts and the end of the cerci measured along the dorsal surface) and wingpad length to the nearest $0.1 \mathrm{~mm}$ with vernier-scale callipers. Using regression analysis we determined the scaling coefficient (reported as the slope of the line) for head width and body length. Size ranges of late instar classes were determined from the relationship between wingpad length and head width. Year class separation was analysed using wingpad to head width relationships together with visual assessment of size frequency distributions (after Ferreras-Romero and Corbet 1999). The level of significance was set at $P=0.05$.

\section{Sediments}

The habitat associations of different size classes of larvae were characterized from August 2002 through April 2003. Sediment samples were collected at each point of larval capture and sieved into six size fractions $(63 \mu \mathrm{m}, 250 \mu \mathrm{m}, 500 \mu \mathrm{m}, 1 \mathrm{~mm}, 2 \mathrm{~mm}, 4 \mathrm{~mm})$. Each sediment fraction was air-dried and weighed, then ashed at $550^{\circ} \mathrm{C}$ for $2 \mathrm{~h}$ and reweighed to obtain weights of both inorganic and organic fractions. Cumulative percent curves were used to determine the median particle size of each sample. We used linear regression to investigate the data for relationships between larval head width, median sediment size and organic fraction. 
A

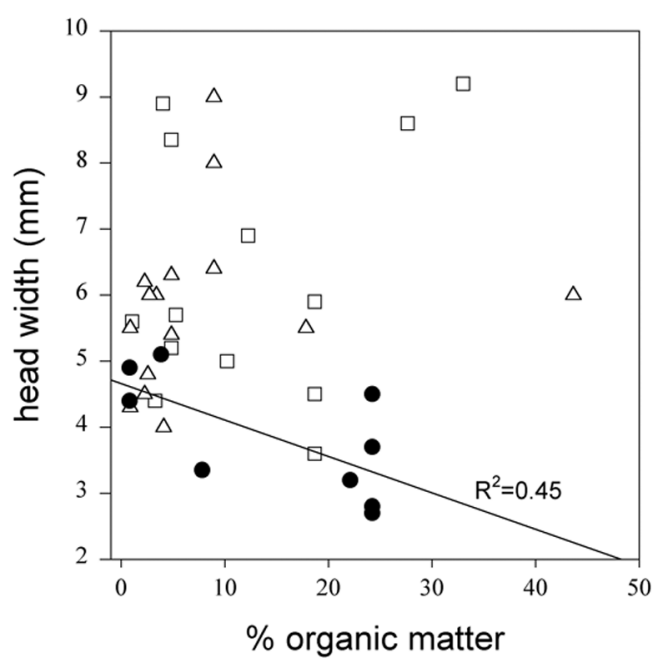

B

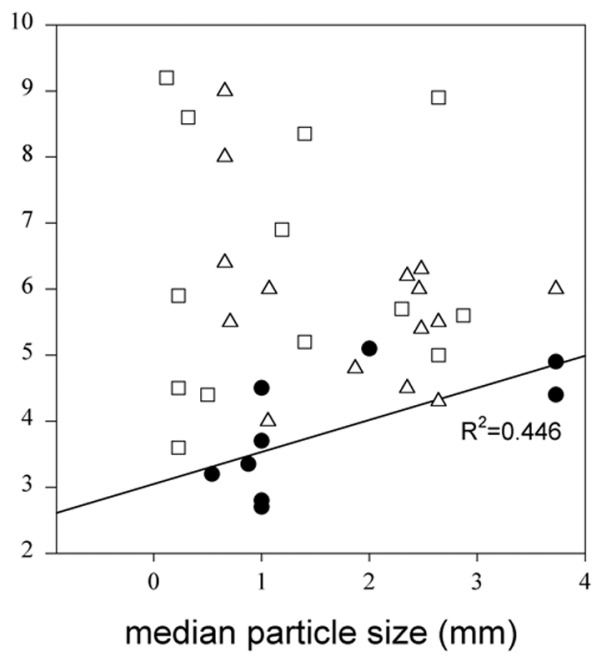

FIGURE 2. Relationship between larval head width of Cordulegaster dorsalis and sediment characteristics - (A) percentage of organic matter; and (B) median particle size. Regressions were significant for F Creek (closed circles) but not for G Creek (open squares) or I Creek (open triangles).

\section{Results and Discussion}

During August 2003 when sample sizes were largest, head width of F-0 (7.5 mm - 9.2 mm) and F-1 (6 $\mathrm{mm}-7.8 \mathrm{~mm}$ ) larvae overlapped, but wingpad lengths were discrete $(3 \mathrm{~mm}-5.5 \mathrm{~mm}$ in F-2, $6.9 \mathrm{~mm}$ $-10 \mathrm{~mm}$ in F-1 and $11.9 \mathrm{~mm}-15.5 \mathrm{~mm}$ in F-0). Total body length and head width were correlated $\left(R^{2}=0.86\right.$, $P<0.001$, TBL $=4.49 \mathrm{HW}-0.59$ ).

In G Creek, larvae of Cordulegaster dorsalis - derived from eggs that likely hatched in early summergrew through late summer and early autumn (Figure 1). At the beginning of their second year most larvae had a head width between $2 \mathrm{~mm}$ and $5 \mathrm{~mm}$. At the end of their second year of growth, larvae achieved headwidths between $5 \mathrm{~mm}$ and $8 \mathrm{~mm}$. Emergence occurred at head widths greater than ca. $8 \mathrm{~mm}$ during July and August of the third year.

The pattern of development that we observed in $\mathrm{G}$ Creek (where sample size was greatest, $n=180$ ) indicated that, in general, $C$. dorsalis larvae require three years to achieve metamorphosis. It is possible to interpret these data as evidence of a split-cohort in the second year with a "fast" group of larvae emerging following accelerated late summer to early fall growth at the end of their first year (Figure 1, dashed line). This interpretation would suggest a winter critical size of ca. $6 \mathrm{~mm}$ (Hdw; Figure 1). Sample sizes were too small in I Creek $(n=30)$ and F Creek $(n=21)$ to bolster a split-cohort interpretation; the Hdw of collected larvae generally support a three-year development.

Many Odonata retain a synchronized emergence period through the use of photoperiod cues (e.g. Aoki
1999; Burcher and Smock 2002). Some species of odonates appear to have both "slow" and "fast" components of the same cohort (Martin et al. 1991; Johnson et al. 1995; Ferreras-Romero and Corbet 1999). We present two possible development schedules for C. dorsalis: a split cohort with a "fast" component emerging after two years and a "slow" component requiring three years to complete development. Cordulegastridae are noted for their protracted larval development, a pattern that might be anticipated of shallow burrowers occupying small, cool streams (Corbet 1999). Two years would represent a remarkably short development time within this family, particularly given the known four-year development time for cordulegastrids in northern California (Kennedy 1917). A more complete record of larval growth rates is required before evidence of a slow and fast development in $C$. dorsalis can be dismissed; in the meantime, the data most strongly support a simple three-year development schedule.

During our surveys for larval development, we observed that small pools seldom contained both large and small larvae. Larvae were most abundant at median particle sizes between $1.5 \mathrm{~mm}$ and $2.5 \mathrm{~mm}$. The head width of larvae was negatively associated with percent total organic content in F Creek (Figure 2a; $\left.P<0.01 ; R^{2}=0.451\right)$ but no relationship was evident in either G or I creeks. Similarly, the head width of larvae was positively associated with median particle size in F Creek (Figure 2b; $P<0.05 ; R^{2}=0.446$ ) while no relationship was evident in either $\mathrm{G}$ or I creeks. It should be noted that median particle and percent 
organic matter are not independent measures; there is a negative correlation $(r=-0.699)$. Post-hoc power analysis suggested that these results must be interpreted cautiously (power $=0.5134$ organic; 0.5072 median particle).

All odonate larvae are opportunistic predators that will eat almost any type of prey they can detect and subdue (Burcher and Smock 2002). Since larger larvae will eat smaller larvae when they encounter them, it seems plausible that some form of habitat partitioning occurs to minimize intraspecific competition and cannibalism. How larvae are distributed at the microhabitat scale will largely determine the frequency of their interactions with conspecifics, affecting the probability of agonistic interactions. The presence or absence of other predators, such as fish, may also affect the microdistribution of larvae. Suhling (1999) demonstrated that small larvae of the gomphid, Onychogomphus uncatus (Charpentier), were significantly more abundant in sand where fish predation was also lowest while medium-sized and large larvae were most abundant in gravel and stones, respectively.

In our study, smaller larvae appeared to be associated with finer sediments with a higher organic fraction. There was a trend supporting larval size sorting relative to sediment microhabitats; however, these results must be interpreted conservatively since this pattern was evident in only one of the three streams surveyed and overall sample sizes were low. In previous habitat surveys of this genus, larvae of $C$. bidentata Sélys were most abundant at a mean grain size of $2.04 \mathrm{~mm}$ while $C$. heros Theischinger were most abundant at $2.79 \mathrm{~mm}$ (Lang et al. 2001). This is consistent with our finding that larvae of $C$. dorsalis were most abundant within medium sand sediments with a median grain size between $1.5 \mathrm{~mm}$ and $2.5 \mathrm{~mm}$.

Although $C$.dorsalis larvae prefer smaller sediments as a group, our evidence is inconclusive regarding microhabitat partitioning amongst larval size classes. There are several alternative explanations for the size sorting we observed between small pools. Larvae may become trapped in these shrinking habitats in a nearly random fashion with respect to size, and the apparent sorting occurs post hoc through intraspecific predation. Smaller larvae would only survive in pools that have a low proportion of late instar larva when these habitats become isolated. Alternatively, the observed size sorting of large and small larvae may relate to a factor that is strongly correlated with sediment grain sizes such as current speed or oxygen levels.

\section{Acknowledgments}

Robert Cannings provided helpful commentary and review. The authors would like to thank Trent Hoover and Conan Phelan for assistance with fieldwork, and members of the Stream and Riparian Research (StaRR) lab at the University of British Columbia for comment. The authors acknowledge funding support from NSERC and the Forest Investment Initiative Account.

\section{Literature Cited}

Aoki, T. 1999. Larval development, emergence and seasonal regulation in Asiagomphus pryeri (Selys) (Odonata: Gomphidae). Hydrobiologia 394: 179-192.

Burcher, C. L., and L. A. Smock. 2002. Habitat distribution, dietary composition and life history characteristics of odonate nymphs in a blackwater coastal plain stream. American Midland Naturalist 148: 75-89.

Cannings, R. A. 2002. Introducing the dragonflies of British Columbia and the Yukon. Royal British Columbia Museum, Victoria.

Corbet, P. S. 1999. Dragonflies: behaviour and ecology of Odonata. Cornell University Press, New York.

Ferreras-Romero, M., and P. S. Corbet. 1999. The life cycle of Cordulegaster boltonii (Donovan, 1807) (Odonata: Cordulegastridae) in the Sierra Morena Mountains (southern Spain). Hydrobiologia 405: 39-48.

Johnson D. M., T. H. Martin, M. Mahato, L. B. Crowder, and P. H. Crowley. 1995. Predation, density dependence, and life histories of dragonflies: A field experiment in a freshwater community. Journal of the North American Benthological Society 14(4): 547-562.

Kennedy, C. H. 1917. Notes on the life history and ecology of the dragonflies (Odonata) of central California and Nevada. Proceedings of the U.S. National Museum 52: 483-635.

Kiffney, P. M., J. S. Richardson, and J. P. Bull. 2003. Responses of periphyton and insects to experimental manipulation of riparian buffer width along forest streams. Journal of Applied Ecology 40: 1060-1076.

Lang, C., H. Müller, and J. A. Waringer. 2001. Larval habitats and longitudinal distribution patterns of Cordulegaster heros Theischinger and C. bidentata Sélys in an Austrian forest stream (Anisoptera: Cordulegastridae). Odonatologica 30: 395-409.

Martin, T. H., D. M. Johnson, and R. D. Moore. 1991. Fishmediated alternative life-history strategies in the dragonfly Epitheca cynosura. Journal of the North American Benthological Society 10: 271-279.

Naiman, R. J., R. E. Bilby, and P. A. Bisson. 2000. Riparian ecology and management in the Pacific coastal rain forest. BioScience 50: 996-1011.

Suhling, F. 1999. Effects of fish on the microdistribution of different larval size groups of Onychogomphus uncatus (Odonata: Gomphidae). Archiv für Hydrobiologie 144: 229-244.

Received 14 September 2005

Accepted 15 March 2007 